Harold A. McAlister and William I. Hartkopf (eds.)

\title{
The Analysis of HST Fine Guidance Sensor Transfer Functions
}

\author{
M. G. LATTANZI, ${ }^{1,2}$ B. BUCCIARELLI, ${ }^{1}$ S. T. HOLFELTZ, \& \\ L. G. TAFF \\ Space Telescope Science Institute, 3700 San Martin Drive, \\ Baltimore, MD 21218, USA
}

\begin{abstract}
We present an overview of the ST ScI calibration program and the reduction, smoothing, and analysis capabilities we have developed to deal with Transfer Functions. The Transfer Function (TF) is the name for the fringe visibility curve produced by the Koesters prism interferometer in the Fine Guidance Sensors (FGSs). The TF for a single, point, monochromatic source becomes modified - in different ways - when we observe a real source with a typical stellar energy spectrum, a finite disc, or a double system. Thus, we have constructed an in depth calibration program and designed and developed a comprehensive set of TF analysis algorithms. The ST ScI calibration program includes the acquisition of reference TFs at 19 different places in the astrometer FGS (there are field-dependent aberrations beyond the wellpublicized spherical aberration of the primary mirror). In addition, for Cycle 2, both stars with finite angular diameters and minor planets will be observed. The software to implement our correlation-based analysis techniques has been extensively tested on artificial binary stars created by combining real TFs and re-introducing photon noise. A wide variety of synthetic binaries - with separations from 10 to 135 milli-arcseconds and magnitude differences from 0 to $4.2 \mathrm{mag}$ - were used so that we can objectively and quantitatively characterize the capability of both the software and the instrument to resolve close pairs and to detect faint companions. Separations to a few milli-arcseconds, position angles to $0: 1$, and magnitude differences to $\pm 0^{\mathrm{m}} 25$ are attainable for primaries brighter than $V=13 \mathrm{mag}$ and $\Delta m<3 \mathrm{mag}$. The software is being incorporated into IRAF/STSDAS at the ST ScI.
\end{abstract}

\section{INTRODUCTION}

How is a binary star observation performed with the Fine Guidance Sensors (FGSs) of the HST? Under the control of the spacecraft's Pointing Control System the astrometer FGS $5^{\prime \prime} \times 5^{\prime \prime}$ instantaneous field-of-view is swept back and forth across the program star. These sweeps are typically $1^{\prime \prime}$ long. From the data acquired during one sweep a 'Transfer Function' can be computed. The Transfer Function (TF) is the name for the fringe visibility generated by the Koesters prism interferometer inside an FGS: As the light bundle traverses the FGS optical system it encounters a $50-50 \%$ beam splitter. The two exit beams are linearly polarized in orthogonal directions. Hence, we speak of the $x$ and $y$ axes within an FGS. Each beam is next sent through a Koesters prism and thence to photomultiplier tubes for photon detection. If we denote the counts, on (say) the $y$-axis, as $A_{y}$ and $B_{y}$ from the two output channels of the prism, then the $y$-axis TF $\left(S_{y}\right)$ is given by, with instrumental and scientific corrections

\footnotetext{
${ }^{1}$ On leave from Torino Observatory.

${ }^{2}$ Affiliated with the Astrophysics Department, Space Science Division, ESA.
} 


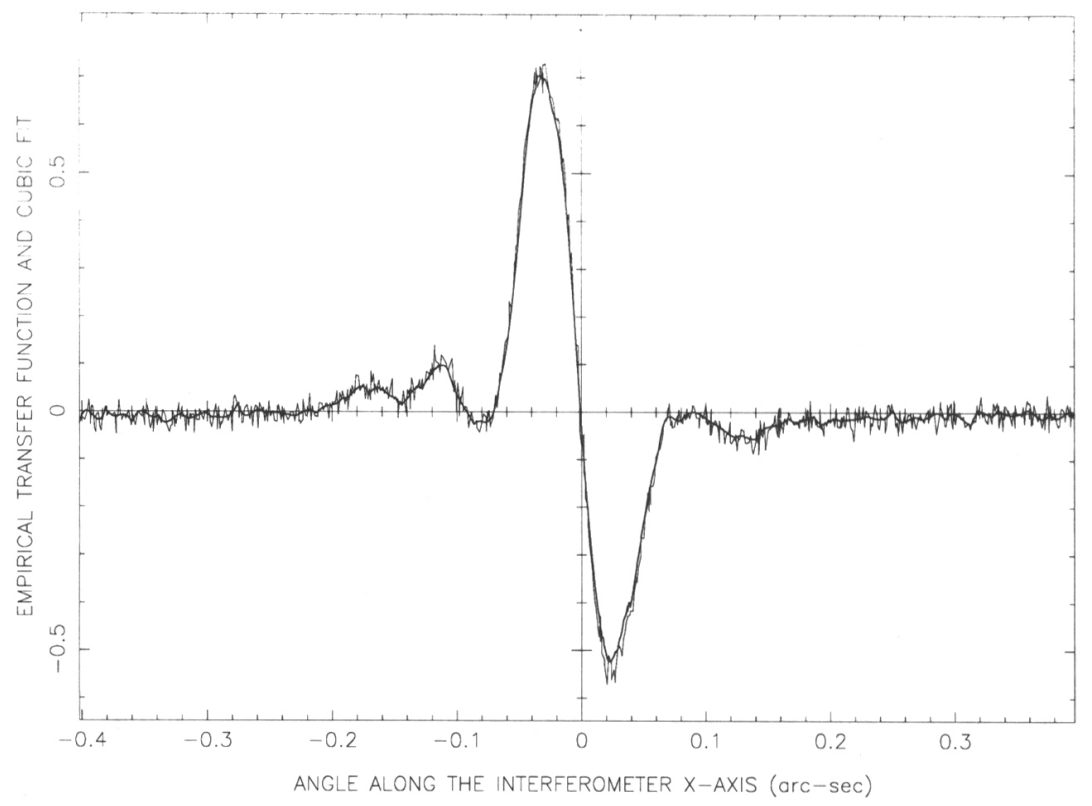

FIGURE 1. High quality, high signal-to-noise $(V=9.6 \mathrm{mag})$, empirical Transfer Function with a smoothed, cubic fit superimposed. The piece-wise cubics only had to satisfy the constraint of continuity.

such as photomultiplier tube mismatch, star selector encoder angle systematic errors, velocity aberration, and so on understood,

$$
S=\left(A_{y}-B_{y}\right) /\left(A_{y}+B_{y}\right)
$$

The expectation for a point source, monochromatic, single star TF was $S(z)=$ $\sin ^{2} z / z[z=2 \pi R \theta / \lambda$ where $R$ is the radius of the primary mirror of the Optical Telescope Assembly (OTA), $\lambda$ is the wavelength, and $\theta$ is the angle from the point of perfect interference]. A real TF is shown in Figure 1.

From Figure 1 we could predict several regions of binary star separation $(\Delta x)$ and primary-secondary magnitude difference $(\Delta m)$ space wherein one would have difficulty accurately determining $\Delta x$ and $\Delta m$. It is expected that these regions - such as small $\Delta x$ and $\Delta m$, or $\Delta x \simeq$ primary to secondary interference fringe distance - will occupy more area in the $\Delta x--\Delta m$ space because of the OTA aberrations. Another effect that could further broaden these regions is the spacecraft jitter induced by the thermally stressed solar array panels. Two different TF jitter suppression algorithms, one discussed elsewhere at this meeting (Franz et al.) and one presented in Franz et al. (AJ, 103, 190, 1992) have been used, with some success, to overcome this additional complication. 


\section{BINARY STAR TRANSFER FUNCTIONS}

How is a binary star Transfer Function (TF) created by the fitting software? Taking the combined OTA/FGS optical system to be a linear one, a binary star TF is a linear combination of two single star TFs. This combination involves a shift corresponding to the separation $\Delta x$ and a diminution of the amplitude of the secondary's fringe visibility function by $10^{-0.4 \Delta m}$. Thus, if $S(y)$ is the $y$ axis single star reference $\mathrm{TF}$, then the $y$ axis binary star TF $B(y)$ is

$$
B(y)=\left[S(y)+10^{-0.4 \Delta m} S(y+\Delta y)\right] /\left(1+10^{-0.4 \Delta m}\right) .
$$

Equation (2) contains several implicit assumptions including that the single star reference TF is from a star of the same color index (or more generally spectral type/luminosity class) of both components of the binary. (The prediction of the effect of varying the color index is for a minor change in the slope of the TF at its null.) Equation (2) also assumes that $S(y)$ is from a place very nearby within the FGS field-of-view to where the program star observations were performed. The empirical TF rapidly changes shape with field angle, hence it is critical to use a local reference for analysis. Thus, the ST ScI calibration plan calls for observations at 19 places within the astrometer FGS. Third Equation (2) assumes that the $x$ and $y$ axes are rigidly fixed. However, $(0,0)$ was at one place for $S(y)$ and at another when the binary star observations were performed. Therefore, $S$ in Equation (2) should be a function of another additive parameter, a translation or offset $y_{0}$. Hence, the complete binary star analysis problem is not three-dimensional (i.e. $\Delta x, \Delta y$, and $\Delta m$ ) but five-dimensional (i.e. $\Delta x, \Delta y$, $\Delta m, x_{0}$, and $\left.y_{0}\right)$. More generally one could attach an $x$ or $y$ subscript to the apparent magnitude difference $\Delta m$ for it can be separately and independently determined on the two axes. The rigorous method is to force $\Delta m_{x}$ to be equal to $\Delta m_{y}$.

Indeed, if you think of the way that TRANSfer Mode observing will typically be performed - with several sweeps of the program star being acquired one after the other as the FGS instantaneous field-of-view is driven back and forth across the binary-offsets are required at the very beginning of the data analysis process to improve the signal-to-noise ratio by 'adding' successive sweeps.

\subsection{Transfer Function Smoothing}

The empirical curve (Figure 1) is fit piecewise to low-order polynomials via a constrained, non-equally weighted, least-squares algorithm. As this is a photon (i.e. Poisson) process we know the standard deviations of each value of $S$ and can determine the weights. The polynomials - linear, quadratic, or cubic are forced to obey continuity conditions at their joining points (knots in spline terminology). In addition, depending on the degree of the polynomial, we may also demand differentiability and even a higher-order degree of smoothness. The constraints are enforced by Lagrange multipliers acting at the joining points.

The above discussion is formal and not the numerically most efficacious way to proceed. The difficulty, see Equations (3) or (4), is in the increase in the number of parameters. Each Lagrange multiplier has to be computed too - even though they are of no real interest. As more parameters are added to the constrained least-squares function, the statistical precision of each of them 
decreases because the number of degrees of freedom is decreasing. Therefore, instead of solving for the Lagrange multipliers, we actually enforce the constraint conditions as additional equations of condition but we use high weights (typically $\left.10^{6}: 1\right)$.

There are two refinements. The choice of the mean width of the intervals is governed by a minimum number of pairs of photomultiplier tube counts constraint. This ensures an adequate number of data points to use to fix the local polynomial's coefficients. The other is that the upper limit for a particular interval's width was to be governed by the curvature of the empirical TF. This was to ensure that we faithfully followed real, short wavelength, features in the data. However, because we are using low-order polynomials and the curvature depends on the second derivative, this has proven difficult to robustly and automatically implement. The piecewise cubic continuous fit is also shown in Figure 1.

The analytical details are as follows: Define $H(x)$ to be the usual Heaviside function; $H(x)=1$ if $x \geq 0,0$ otherwise. So, if $a<b$ then $h(x ; a, b)=$ $H(x-a) H(b-x)$ is unity for $x \in[a, b]$ and zero otherwise. Next define $N$ joints $z_{1}, z_{2}, \ldots, z_{N}$ where we will enforce continuity, smoothness, and so on. We also need the general polynomial of degree $d ; P(x)=a+b x+c x^{2}+\cdots+q x^{d}$. We need one $d$ 'th order polynomial per interval (the boundaries of the intervals are defined by the set of joint locations $\left\{z_{n}\right\}$ ), so we use a double indexing scheme for the coefficients in $P=P_{n}(n=0,1, \ldots, N) ; P_{n}(x)=a_{n 0}+a_{n 1} x+a_{n 2} x^{2}+\cdots+a_{n d} x^{d}$. $P_{n}$ is defined everywhere but will only be used on $\left[z_{n}, z_{n+1}\right]$.

We want to represent a function $F(x)$ on $x \in\left[x_{\min }=z_{1}, x_{\max }=z_{N}\right]$ by a linear combination of $N-1 d^{\prime}$ th order polynomials $\left\{P_{n}\right\}$ which obey various boundary conditions at $\left\{z_{n}\right\}$. Thus, we write

$$
F(x)=\sum_{n=1}^{N-1} h\left(x ; z_{n}, z_{n+1}\right) P_{n}(x)
$$

where, were we only enforcing continuity at the $N-2$ interior points $\left\{z_{n}\right\}$ one would have the constraints $P_{n}\left(z_{n+1}\right)=P_{n+1}\left(z_{n+1}\right) ; n=1, \cdots, N-2$. We determine the $\left\{a_{n m}\right\}$ based on least-squares given that we have measured $F(x)$ at $x_{\min }=x_{1}, x_{2}, \ldots, x_{L}=x_{\max }$. Thus, we form

$$
\begin{aligned}
R_{c}=\sum_{\ell=1}^{L} w_{\ell} & {\left[F\left(x_{\ell}\right)-\sum_{n=1}^{N-1} h\left(x_{\ell} ; z_{n}, z_{n+1}\right) P_{n}\left(x_{\ell}\right)\right]^{2} } \\
& -\sum_{n=1}^{N-2} \lambda_{n+1}\left[P_{n}\left(z_{n+1}\right)-P_{n+1}\left(z_{n+1}\right)\right]
\end{aligned}
$$

and minimize $R_{c}$ with respect to the $(N-1) d$ values of $\left\{a_{n m}\right\}$ and the $N-2$ Lagrange multipliers $\left\{\lambda_{n}\right\}$. The weights $w_{\ell}$ are the photon-noise implied weights of the TF from Equation (1). There is a point of subtlety for $A$ and $B$ should be perfectly anti-correlated; thus $\sigma_{S}=(\sqrt{A}+\sqrt{B}) / C, w_{S}=1 / \sigma_{S}^{2}$. (Correlation coefficients from real data are typically less than -0.9 .)

The extension to incorporate smoothness at the joints is accomplished by 
minimizing $R_{\bullet}\left[P_{n}^{\prime}\left(z_{k}\right)\right.$ means $\left.\left.\frac{d P_{n}(x)}{\Delta x}\right|_{x=z_{k}}\right]$,

$$
R_{\AA}=R_{c}-\sum_{n=1}^{N-2} \Lambda_{n+1}\left[P_{n}^{\prime}\left(z_{n+1}\right)-P_{n+1}^{\prime}\left(z_{n+1}\right)\right] .
$$

\section{BINARY STAR TRANSFER FUNCTION ANALYSIS}

\subsection{Least-Squares Approaches}

The least-squares approach is to compute the difference between the observed $(O)$ quantities and their computed values $[C=B$ from Equation (2)] i.e. $O-C$, square, weight, sum them, and then try and minimize the sum of the squares with respect to the three parameters $x_{0}, \Delta x$, and $\Delta m_{x}$. Because $C$ depends implicitly on the unknowns, no straightforward solution technique is going to work well. One has to perform a linearization around the current guess for the solution, compute new corrections using first-order Taylor series, and so on. Such iteration procedures are not robust - they typically need good, sometimes excellent, starting values. Attempts to assist the numerical process, by, for instance, analytically calculating higher derivatives to use a steepest descent technique, can represent a large amount of work because of the non-linear nature of the problem. Hence, we decided not to further pursue this course.

However, a 'brute force' least-squares algorithm can be easily constructed. The interesting volume of $x_{0}, \Delta x$, and $\Delta m_{x}$ space is $[-500,500] \times[-500,500] \times$ $[0,4.5]$, where the units for $x_{0}$ and $\Delta x$ are milli-arcseconds (mas) and those for $\Delta m_{x}$ are magnitudes. The entire space could be explored over a multidimensional grid using the sum of the squares defined above as the measure of success. Thus, the following scheme:

1. Center the search region.

2. Define initial search lengths $L\left(x_{0}\right), L(\Delta x)$, and $L(\Delta m)$.

3. Define initial search steps $D\left(x_{0}\right), D(\Delta x)$, and $D(\Delta m)$.

4. Explore the ranges $2 L\left(x_{0}\right), 2 L(\Delta x)$, and $2 L(\Delta m)$ using step sizes $D\left(x_{0}\right)$, $D(\Delta x)$, and $D(\Delta m)$ respectively by computing the weighted sum of the squares at $\left[2 L\left(x_{0}\right) / D\left(x_{0}\right)+1\right][2 L(\Delta x) / D(\Delta x)+1]\left[2 L\left(\Delta m_{x}\right) / D\left(\Delta m_{x}\right)+1\right]$ places.

5. Find the global minimum, over this grid, of the weighted sum of the squares of the $O-C$ 's.

6. Place the new search center at this location.

7. Decrease the current search ranges and current step sizes by a factor of 2 (say).

8. Repeat steps $4,5,6$, and 7 until the step sizes are driven to an agreed upon minimum; for instance 1 mas and 0.05 mag.

There is one necessary criterion; the solution must always lie within the new search volume. If, the earlier search steps were too large or the sum of the squares surface has a lot of structure, we fix on a secondary minimum, then this algorithm will converge, with high precision, to an inaccurate answer. Ensuring that this does not happen brings us back to one of the objections we 
brought up with regard to the non-linear least-squares method. However, in this case, there is a purely computational insurance policy available - we just use values of the step sizes so small that the global minimum must be encountered. The real advantage of this technique is, that given a good initial guess, so that both the starting volume and the original step sizes can be relatively small, the right answer will be accurately obtained very quickly. Hence, we searched for a computationally efficient algorithm which could provide this initial information in a robust fashion. The one we have used is described in the next subsection. Another idea (Hershey 1992, PASP, in press), based on Fourier Transforms and an analog with deconvolution techniques, holds promise for providing initial guesses more rapidly.

\subsection{Correlation Integral Approaches}

The model in Equation (2) is fitted to the observed TF and the parameters $\Delta x$ and $\Delta m_{x}$ derived. As before, this is performed by generating a grid of models by varying $\Delta x, x_{o}$, and $\Delta m_{x}$. Each model is cross-correlated with the observed TF by computing the correlation integral

$$
c(x)=\int B(t-x) O(t) d t .
$$

The ideal binary star function $B$ is cross-correlated with the empirical Koesters prism fringe visibility function $O$. The sought-for value of $x, x_{0}$, such that

$$
c\left(x_{o}\right)=\text { maximum }
$$

represents the shift along this axis between the two functions as discussed in the previous section.

Next, the best-fit model is determined as the one with parameters which minimize the sum of the squares of the differences between $B$ and $O$, viz.

$$
\int\left[B\left(t-x_{0}\right)-O(t)\right]^{2} d t=\text { minimum. }
$$

The polynomial smoothing not only increases the resolution of the subsequent cross-correlation but makes it possible to compute the correlation integral $c$ in Equation (5a), as well as the sum of squares, to be minimized in Equation (6), analytically. The accuracy of the fit can be improved, now using the answers from the correlation technique as initial guesses for the final least-squares adjustment. All possible options of correlation/least-squares fitting are currently supported in our software.

\section{NUMERICAL TESTING}

There are several different issues that have to be treated. First, given that we have a set of reference curves, and given that we have noisy observational data, can our method(s) deduce the correct answers for the triplet of offset $\left(x_{0}\right)$, separation $(\Delta x)$ and magnitude difference $\left(\Delta m_{x}\right)$ ? Can they do so without the necessity of providing them with a reasonably close initial guess (that is do they 
have global convergence properties)? Can they do so with good accuracy (that is can they find the correct solution set)? Second, is there repeatability? In particular, if the same binary system is repeatedly observed, will any of our methods converge to the same solution set? If not the identical solution set, then what is the dispersion of the results (as this determines the true precision of the algorithm)? Can we characterize the standard deviations of the results in a simple way in terms of the signal-to-noise ratio? Third, are there values of offset, separation, or magnitude difference which these techniques can not adequately deal with? If so, how does the precision decrease as the edge of the resolvability surface in offset/separation/magnitude difference space is approached? What role does the total magnitude of the binary play in all this? Finally, although our concentration in this paper is on binary stars, can the same software be used for triple star analysis? Angular diameter analysis? Does it have the same global convergence properties? Repeatability properties? and so on.

In this section we describe the beginning of an extensive set of numerical experiments we are performing to thoroughly and completely answer these questions.

First we wanted to demonstrate convergence of the correlation technique in a global sense. We also wanted to show that the least-squares algorithm, commencing from the correlation answer, could then rapidly approach the true answer. In all instances the offset (i.e. $x_{0}$ ) is a minor issue. We have developed a separate method designed to find an approximate value of the offset which the correlation technique then refines. It does so by exploiting a rough value for the null of the TF leaving, typically, \pm 10 mas uncertainty. Thus, while we shall report on the results for the offset, we shall not further dwell on them.

The correlation part of the software looks for the minimum in Equation (6) integral can attain by searching through $\Delta x$ and $\Delta m$ space with a fixed (an arbitrary) spacing. For each $\Delta x, \Delta m$ pair, Equation (5b) is first satisfied with respect to $x_{0}$. Typically we use a value of 5 mas for $\Delta x$ and $0.1 \mathrm{mag}$ for $\Delta m_{x}$. Therefore, using a test value for the separation or magnitude difference that is an integral multiple of these spacings would be unconvincing. A matrix of ten separations $(\Delta x=10.2,27.9,38.4,45.7,64.9,79.9,81.6,103.0,124.8$, and $135.1 \mathrm{mas})$ and ten magnitude differences $(\Delta m=0.000,0.234,0.456,1.567$, $2.222,2.617,2.987,3.210,3.540$, and $4.170 \mathrm{mag}$ ) were chosen. They have been slightly weighted towards zero separation, zero magnitude difference, and large magnitude differences as these form the stressing cases. For these 100 values the full software set was allowed complete freedom with respect to the initial values. The results of the intermediate correlation results and the final least-squares results are given in Figures 2 and 3. By and large the true values are faithfully reproduced.

The errors are largest, in $\Delta x$ and $\Delta m_{x}$ space, where we already knew there would be problems. The difficulty with fainter secondaries is a lack of signalto-noise ratio in the TFs compounded by a less than perfect form for the single star curves and spacecraft jitter. As the separation between the pair approaches zero, these same confounding issues limit our ability to tell that there are in fact two stars there as opposed to a photon-noise deformed, single-star TF (of $V=12.7 \mathrm{mag}$ in these cases). Results for different magnitude primaries (9.6 and $14.8 \mathrm{mag}$ ) and for Monte Carlo simulations involving photon noise will be reported on over the course of time. 


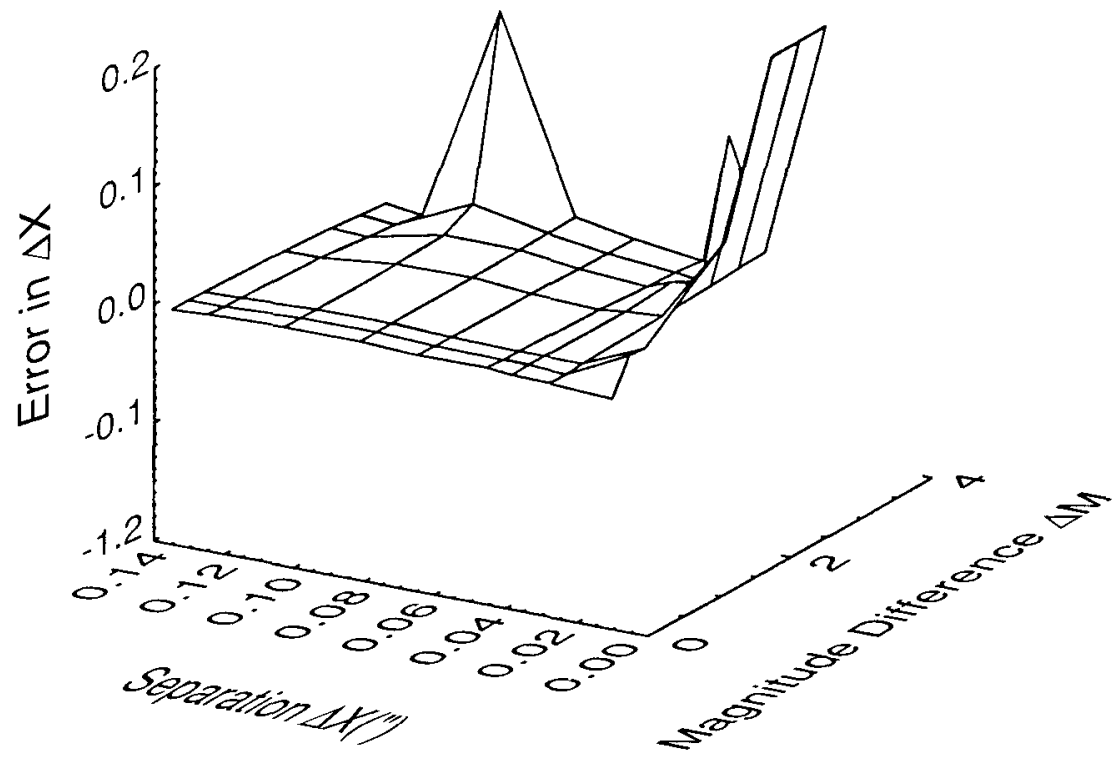

FIGURE 2. Errors in separation $(\Delta x)$ in arc seconds as a function of separation and magnitude difference for a $V=12.7 \mathrm{mag}$ primary. Note the poor results for zero separation and for the faintest secondaries when the separation is equal to the primary/secondary peak difference in the TF.

\section{SUMMARY}

This paper has reported on the first realistic simulations of the precision and accuracy of binary star reconstruction from the Fine Guidance Sensor TRANSfer function observing mode. We expect to be able to retrieve a few milli-arcseconds for the separation for separations beyond $\sim 10-15$ mas, a tenth of degree for the position angle for similar separations (which is partially dependent on the reconstructed attitude of the spacecraft), and a few tenths of a magnitude for the primary/secondary magnitude difference for magnitude differences less than $3 \mathrm{mag}$. All of this refers to systems wherein the primary is brighter than $14^{\text {th }}$ magnitude. This level of performance will deteriorate with increasing magnitude difference, decreasing separation, and decreasing magnitude of the primary.

\section{ACKNOWLEDGMENTS}

This work is partially based on observations with the NASA/ESA Hubble Space Telescope obtained at the Space Telescope Science Institute. ST ScI is operated by AURA, Inc. under contract NAS5-26555 to NASA. Work also supported in part by NASA grant NAGW-2597. 


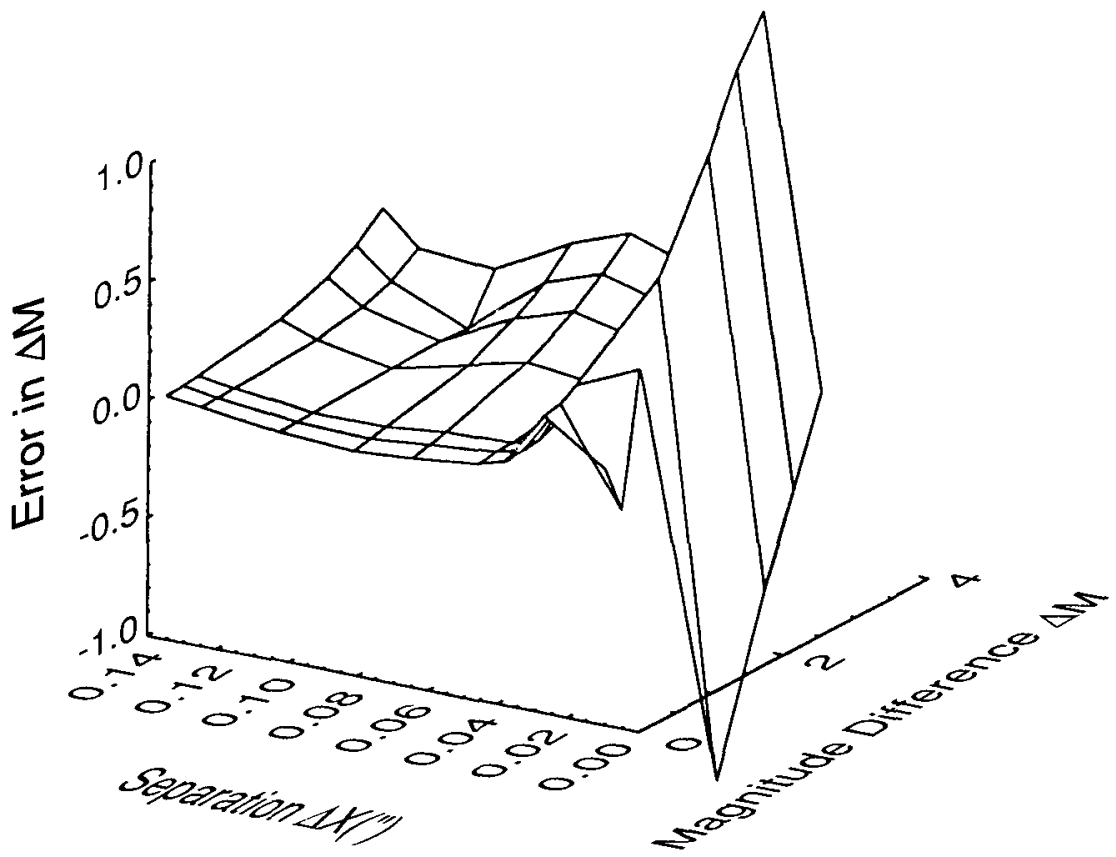

FIGURE 3. As in Figure 2 but errors in the magnitude difference.

\section{DISCUSSION}

FRANZ: The fact that the "bump" due to a binary component may coincide with the secondary "bump" in the transfer function is not necessarily of practical importance. You observe the object at a different roll angle and thus at a different projected separation, avoiding the coincidence.

TAFF: This is absolutely correct for a binary with a known orbit. However, I still hope that the HST Telescope Allocation Committee will grant sufficient observing time to the FGS's to go on a "fishing" expedition for binary stars. Then this becomes a consideration.

MCALISTER: Do you have any way to externally calibrate $\Delta \mathrm{m}$ to add confidence to your simulations?

TAFF: There is a separate photometric calibration of the FGS's. This should produce a $\pm 0.01 \mathrm{mag}$ calibration for photometry. Calibrating $\Delta \mathrm{m}$ will come from the Monte Carlo simulations I alluded to and this will be much larger, $\pm 0.3 \mathrm{mag}$ at $\Delta \mathrm{m}=1.5 \mathrm{mag}$. 